\title{
Moving from Association to Causation: Deciphering the Role of the Gut Microbiota in Hypertension
}

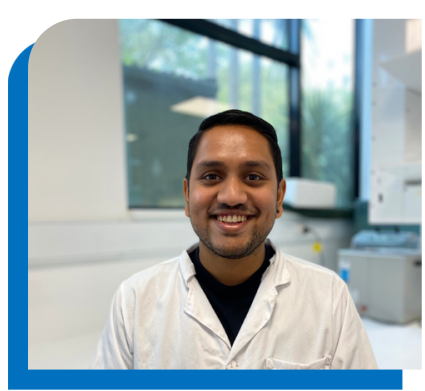

\author{
RIKEISH R. MURALITHARAN
}

Hypertension Research Laboratory, School of Biological Sciences, Monash

University, Australia

\section{DOI:10.30824/2110-16}

The gut harbours trillions of microbes including bacteria, fungi, and viruses, collectively known as the gut microbiota. These microbes interact among themselves, but also with the host's immune system and environment (e.g. food). There is increasing evidence to support the role of the gut microbiota and its genome, the gut microbiome, in hypertension'. Despite the increasing number of microbe-wide association studies (MWAS), identifying specific causal microbes that contribute to blood pressure (BP) regulation has been challenging ${ }^{2,3}$. This is unsurprising given our experience with genomewide association studies (GWAS), which have identified hundreds of genes with little understanding about their role in BP control per se $\mathrm{e}^{4}$. Considering the gut microbiota is influenced by many intrinsic (e.g., host's genetics ${ }^{5}$ ) and extrinsic factors (e.g. diet, geography, ethnicity, seasonal variations, circadian cycle, and medication use), gut microbiome studies require careful experimental design. This is easier done using laboratory animals than humans ${ }^{6}$.

Instead of identifying causal microbes, a more fruitful approach is to assign functions to microbes to better understand their roles. Through this approach, it was shown some bacteria, such as butyrate-producers, are lacking in hypertensive patients. Indeed, we recently discovered that microbial gene pathways might be more important than specific microbial taxa ${ }^{7}$. This has also led to the discovery of the importance of metabolites such as short-chain fatty acids (SCFAs) in hypertension ${ }^{8}$ and trimethyl-amine oxide (TMAO) in cardiovascular diseases ${ }^{9}$.

To further advance the field, mechanistically linking microbes or metabolites to function and phenotype is essential. There are multiple ways to achieve this, namely a) reverse microbiome approaches, b) forward microbiome approaches, and c) microbe-phenotype triangulation (Figure 1) ${ }^{9}$.

An example of the use of these approaches is a recent publication in Circulation Research, by Dr. David Durgan and his team. They elegantly employed some of these approaches to demonstrate mechanistically how the gut microbiota changes with intermittent fasting and how that influences $\mathrm{BP}^{10}$. Using a forward microbiome approach, they first compared the gut microbiome between their experimental groups and identified differentially abundant bacteria that metabolised bile acid. To confirm this, they performed caecal and plasma metabolomics and found evidence supporting differences in bile acid metabolites. Armed with sufficient evidence, they went on to use a reverse microbiome approach to confirm their findings by dietary supplementation and agonist treatment. By using a combination of these methods, they were able to pinpoint that increased BP in hypertensive rats was a result of reduced plasma bile acid levels which could be restored with either a) intermittent fasting, b) supplementing cholic acid (precursor of bile acids) or c) agonist treatment of TGR5, a bile acid receptor. Although their studies were not validated in the context of human hypertension, a recent clinical study showed fasting influenced the gut microbiome and reduced BP in patients with metabolic syndrome ${ }^{11}$. However, altered bile acid metabolism was not discovered in those patients, potentially because of differences in the models and diet.

Moving forward, a push towards identifying causal links of the gut microbiome in hypertension is necessary to advance the field. Confirmatory experiments need to be performed and validated in other models and ideally in clinical interventional studies. 
References

1. R. Muralitharan R, Marques FZ. Diet-related gut microbial metabolites and sensing in hypertension. Journal of Human Hypertension. 2021;35:162-169

2. Palmu J, Salosensaari A, Havulinna AS, et al. Association between the gut microbiota and blood pressure in a population cohort of 6953 individuals. Journal of the American Heart Association. 2020;9:e016641

3. Louca P, Menni C, Padmanabhan S. Genomic determinants of hypertension with a focus on metabolomics and the gut microbiome. American journal of hypertension. 2020;33:473-481

4. Evangelou E, Warren HR, et al., Million Veteran P. Genetic analysis of over 1 million people identifies 535 new loci associated with blood pressure traits. Nature genetics. 2018;50:1412-1425

5. Grieneisen L, Dasari M, Gould TJ, et al. Gut microbiome heritability is nearly universal but environmentally contingent. Science. 2021;373:181-186

6. Marques FZ, Jama HA, Tsyganov $\mathrm{K}$, et al. Guidelines for transparency on gut microbiome studies in essential and experimental hypertension. Hypertension. 2019;74:1279-1293

7. Nakai M, Ribeiro RV, Stevens BR, et al. Essential hypertension is associated with changes in gut microbial metabolic pathways: A multi-site analysis of ambulatory blood pressure. medRxiv. 2021:2021.2002.2018.21252018

8. Marques FZ, Nelson E, Chu P-Y, et al. Highfiber diet and acetate supplementation change the gut microbiota and prevent the development of hypertension and heart failure in hypertensive mice. Circulation. 2017;135:964-977

9. Muralitharan RR, Jama HA, Xie L, et al. Microbial peer pressure. Hypertension. 2020;76:1674-1687

10. Shi H, Zhang B, Abo-HamzyT, et al. Restructuring the gut microbiota by intermittent fasting lowers blood pressure. Circulation Research. 2021;128:1240-1254

11. Maifeld A, Bartolomaeus H, Löber U, Avery EG, Set al. Fasting alters the gut microbiome reducing blood pressure and body weight in metabolic syndrome patients. Nature communications. 2021;12:1970

\section{a:care congress 20-21 October 2021:}

The first congress exploring how to improve medication adherence through behavioral science.

- Join us to hear from world-renowned experts

- Learn about behavioral interventions and digital technology

- Collaborate with peers

\section{a:care}

THIS IS ADHERENCE REIMAGINED.

This is care empowered.

REGISTER TODAY

acarepro.abbott.com/en/congress 2021

口我田
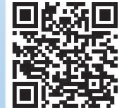

GLO2199177

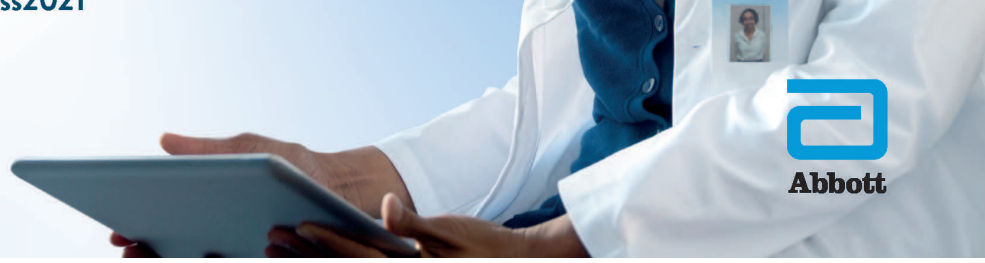

